\title{
Ruptured Aneurysm Arising from the Anterior Choroidal Artery's Cisternal Segment
}

\author{
Nancy McLaughlin, Michel W. Bojanowski
}

Can. J. Neurol. Sci. 2010; 37: 283-285

Aneurysms of the anterior choroidal artery (AChoA) usually arise at the junction of the AChoA and the internal carotid artery (ICA). They account for 2-4\% of intracranial aneurysms ${ }^{1}$. Distal AChoA aneurysms are rare, most originating from the plexal segment. Those arising from the cisternal segment are even rarer. Distal AChoA aneurysms, especially those in relation with the cisternal segment, represent a surgical challenge given the small diameter of the parent vessel. We describe a case of an aneurysm budding from the AChoA's cisternal segment requiring surgical treatment and review the literature.

\section{CASE RePORT}

A 40-year-old female previously in good health, presented with a sudden onset of severe headache associated with nausea and vomiting. Neurological examination was normal on admission. Computed tomography (CT) showed subarachnoid hemorrhage (SAH) in the left ambient cistern and left Sylvian fissure (Figure 1). Routine hematological and biochemical examinations were normal. Cerebral angiography revealed a $3 \mathrm{~mm}$ aneurysm originating from the cisternal segment of the left AChoA (Figure 2A). No other vascular abnormality was documented.

It was felt that embolization was not an option given the high risk of parent vessel occlusion. Within 48 hours of the hemorrhage, a left frontotemporal craniotomy was performed. Under microsurgical magnification, the basal cisterns were opened and the proximal Sylvian fissure was split. The arachnoid along the medial border of the ICA was dissected up to the ICA's bifurcation. After identification of the posterior communicating artery, dissection of the lateral border of the ICA was undertaken. At this point, a multilobulated aneurysm was identified originating from the AchoA, distant from the internal carotid. The proximal carotid being temporarily clipped, the aneurysm collapsed, revealing the course of the AChoA. After dissection of the aneurysm's neck, the aneurysm was clipped, preserving the cisternal segment of AChoA.

The patient was neurologically intact following surgery. No ischemic lesion was seen on the post-operative scan. Postoperative angiography performed nine days following $\mathrm{SAH}$ showed a patent AChoA artery and complete exclusion of the distal cisternal AChoA aneurysm (Figure 2B).

\section{DisCUSSION}

Authors have divided the AChoA into a cisternal and plexal segment $^{2}$ (Figure 3). The cisternal segment extends from the

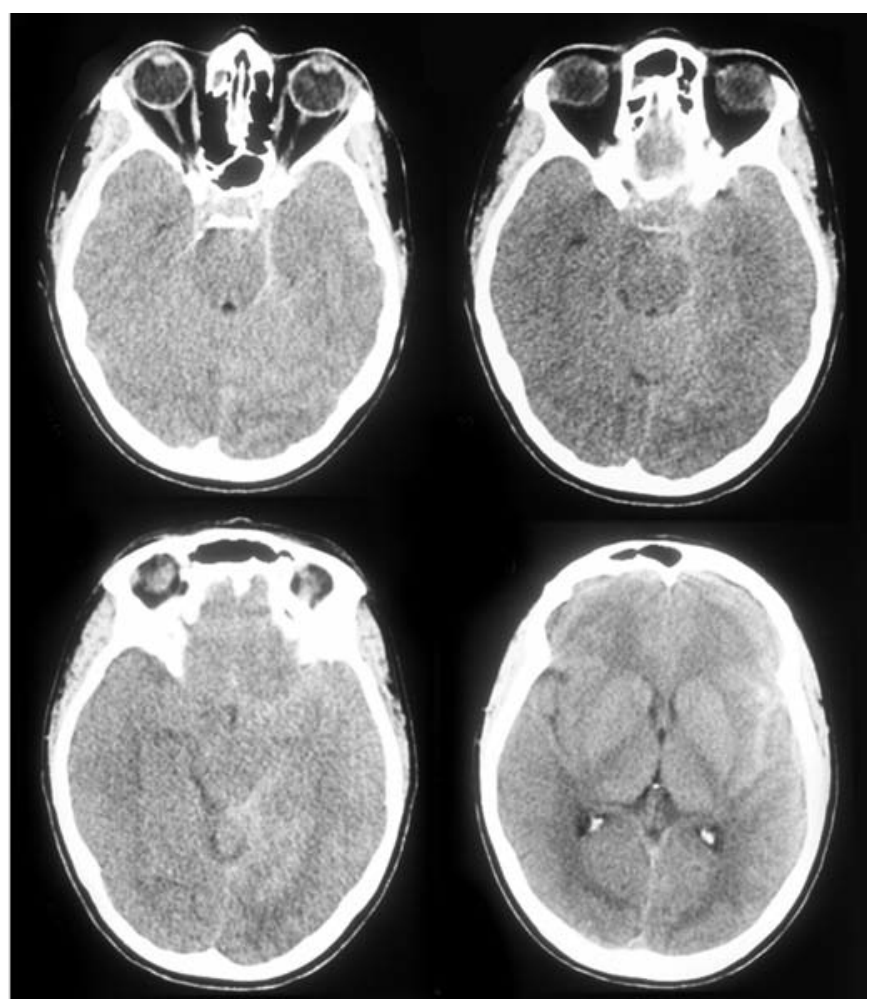

Figure 1: Pre-operative CT scan documenting a subarachnoid hemorrhage located in the left ambient cistern and left Sylvian fissure.

AChoA's origin to the choroidal fissure. Several vital branches of the AChoA originate from the cisternal segment, supplying the posterior two thirds of the posterior limb of the internal capsule, the globus pallidus, the optic tract, the beginning of the optic radiation, the lateral aspect of the lateral geniculate body, the

From the Division of Neurosurgery, Department of Surgery, Centre Hospitalier de l’Université de Montréal - Hôpital Notre-Dame, Montreal, QC, Canada.

Received January 20, 2009. Final Revisions Submitted September 9, 2009. Correspondence to: Michel W. Bojanowski, Neurosurgery Department, CHUMHôpital Notre-Dame, 1560 Sherbrooke Est, Montreal, Quebec, H2L 4M1, Canada. 


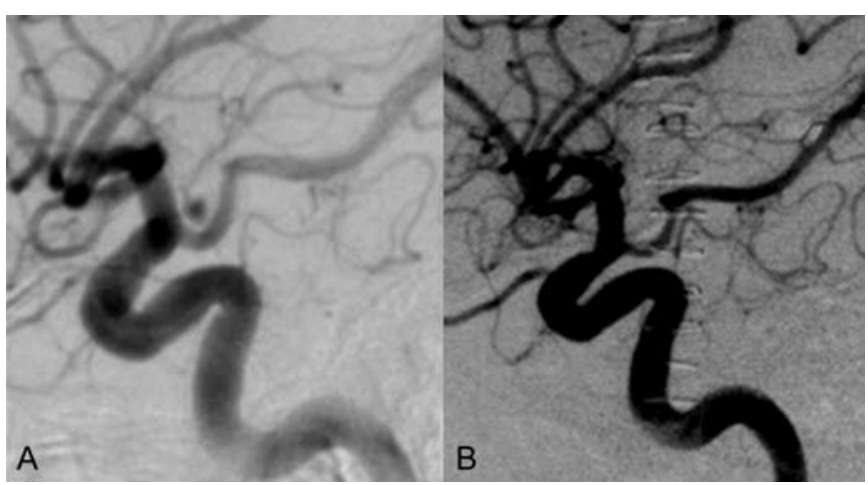

Figure 2: A) Pre-operative angiography revealing the presence of a $3 \mathrm{~mm}$ aneurysm originating from the cisternal segment of the left anterior choroidal artery (AChoA). No other vascular abnormality was present. B) Post-operative angiography showing a patent AChoA artery and complete exclusion of the distal cisternal AChoA aneurysm.

middle third of the crus cerebri, the uncohippocampal region, and the anterior and posterior perforated substance ${ }^{3}$. Consequently, the occlusion of this segment could be associated with severe neurological deficits. On the other hand, the plexal segment extends from the choroidal fissure to the choroid plexus of the temporal horn. Some branches pass posterior into the atrium and then forward above the thalamus to supply the choroid plexus of the lateral ventricles as far forward as the foramen of $\mathrm{Monro}^{2}$. Its occlusion is usually without any significant neurological consequence.

Aneurysms of the anterior choroidal artery aneurysms are mostly located at the AChoA and ICA junction. Rare cases of distal AChoA aneurysms have been reported. Including our case, 34 cases have been described in the literature. Clinical presentation includes $\mathrm{SAH}$, hemorrhage, intraventricular hemorrhage and ischemic symptoms. Distal AChoA aneurysms

Table: Review of the literature on cisternal anterior choroidal aneurysms

\begin{tabular}{l|l|l|l|l}
\hline Author & $\begin{array}{l}\text { Age, } \\
\text { Sex }\end{array}$ & Presentation & Cause & Treatment \\
\hline Hung, 1996 & $35, \mathrm{~F}$ & SAH & Idiopathic & $\begin{array}{l}\text { Clipping, } \\
\text { sacrificing the } \\
\text { superior branch } \\
\text { of the AchoA }\end{array}$ \\
\hline $\begin{array}{l}\text { Matsuura, } \\
2000\end{array}$ & $42, \mathrm{M}$ & Stroke & Dissection & Conservative \\
\hline Ahn, 2006 & $60, \mathrm{~F}$ & SAH, IVH & Infectious & $\begin{array}{l}\text { Planned clipping } \\
\text { but patient died } \\
\text { prior }\end{array}$ \\
\hline $\begin{array}{l}\text { McLaughlin, } \\
\begin{array}{l}2009 \\
\text { (present } \\
\text { case) }\end{array}\end{array}$ & $40, \mathrm{~F}$ & SAH, IVH & Idiopathic & Clipping \\
\hline
\end{tabular}

$\mathrm{SAH}=$ subarachnoid hemorrhage; $\mathrm{IVH}=$ intraventricular hemorrhage

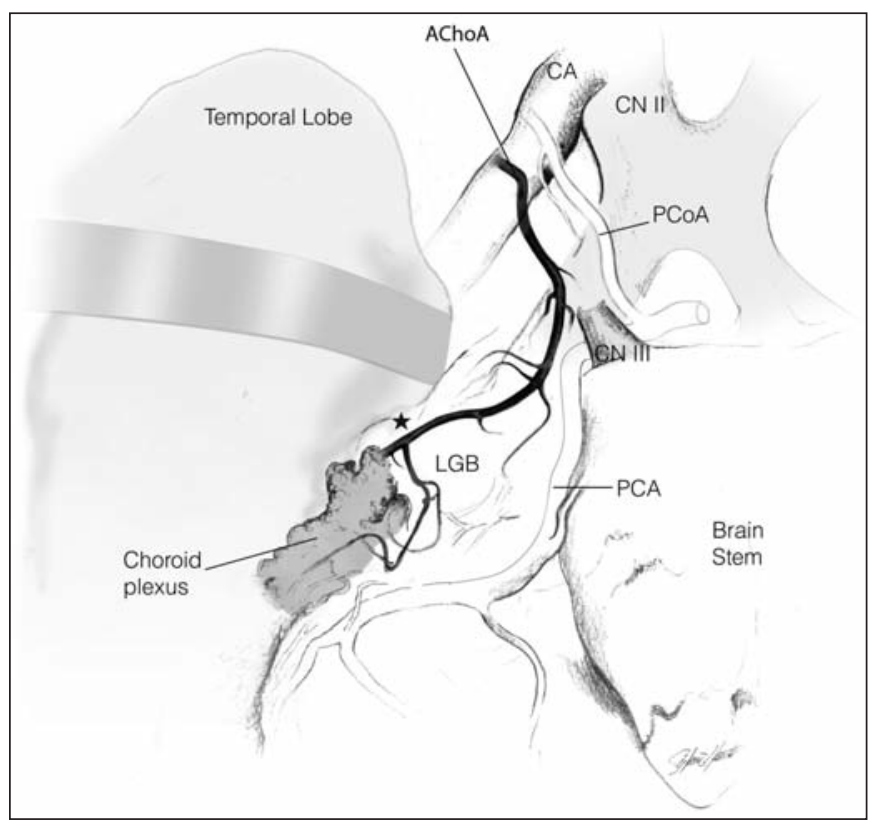

Figure 3: Artist representation of the AChoA, from its origin to its entry in the choroidal fissure to vascularize the choroids plexus. * represents the transition from the distal cisternal segment to the proximal plexal segment. AChoA: anterior choroidal artery; $C N$ : cranial nerve; $C A$ : carotid artery; LGB: lateral geniculate body; PCA: posterior cerebral artery; PcoA: posterior communicating artery.

are frequently associated with the following conditions: moya moya phenomenon, history of trauma, atherosclerosis, arteriovenous malformation. However, almost a third of distal AChoA aneurysms are of idiopathic origin. Only three reported cases of distal AChoA aneurysms are located within the AchoA's cisternal segment. One case of AChoA aneurysm arising directly from the AChoA's cisternal segment ${ }^{4}$ and two cases of aneurysms budding from a branch of the AChoA's cisternal segment ${ }^{5,6}$ have been reported. Including our case, the clinical features, associated pathologies and treatment of these cases are summarized in the Table.

Distal AChoA aneurysms represent a surgical challenge to the neurosurgeon. Due to the small lumen of the parent artery, preservation of its patency while clipping the neck of the aneurysm may be difficult ${ }^{6}$. Indeed, preserving patency in such circumstances might be more challenging than with aneurysms located at the AChoA-ICA junction whose origin partly involves the larger ICA. Furthermore, although trapping of plexal aneurysms usually does not cause a neurological deficit, occlusion of the cisternal segment or some of its branches results most often in neurological deficits of inconstant severity given the presence of collateral circulation through numerous anastomosis with the posterior communicating artery and posterior cerebral artery.

The operative approach for aneurysms of the AChoA's cisternal segment is similar to those located at the AChoA-ICA junction. Preservation of the cisternal AChoA segment is 
imperative given the vital branches that arise from this segment. If the aneurysm buds from a branch of the AChoA's cisternal segment, trapping may be done without any neurological deficits given the abundant collaterals. However, for an aneurysm arising directly from the AChoA, trapping could result in hemiplegia, hemianesthesia and homonyme hemianopsia. For obvious reasons, endovascular treatment may be considered only if direct coiling of the aneurysm is feasible with parent artery preservation.

In summary, aneurysms originating from the cisternal segment of the AChoA represent a rare entity. They represent a therapeutic challenge as it is imperative to preserve the patency of the parent artery.

\section{REFERENCES}

1. Yasargil MG, Yonas H, Gasser JC. Anterior choroidal artery aneurysms: their anatomy and surgical significance. Surg Neurol. 1978;9(2):129-38.

2. Rhoton AL Jr, Fujii K, Fiadd B. Microsurgical anatomy of the anterior choroidal artery. Surg Neurol. 1979;12(2):171-87.

3. Abbie AA. The blood supply of the lateral geniculate body, with a note on the morphology of the choroidal arteries. J Anat. 1933;64 (Pt4):491-521.

4. Matsuura H, Otawara Y, Suzuki M, Ogawa A. Dissecting aneurysm of the anterior choroidal artery: angiographic and MR imaging findings. Surg Neurol. 53(4):334-6.

5. Hung KS, Lee TC. Aneurysm of superior branch of anterior choroidal artery mimicking carotid bifurcation aneurysms. Case report. Acta Neurochir. 1996;138(12):1464-7.

6. Ahn JY, Han IB, Hong CK, Joo JY. Ruptured distal anterior choroidal artery aneurysm. J Clin Neurosci. 2006;13(8):872-5. 